\title{
A New Jammer Suppression Method in MIMO Radar Using Matrix Pencil Method and Generalized Likelihood Ratio Test
}

\author{
Kang-in Lee, ${ }^{1}$ Wonjune Kang, ${ }^{1}$ Young-Seek Chung, ${ }^{1}$ Hoon-Gee Yang, ${ }^{1}$ and Jong Mann Kim ${ }^{2}$ \\ ${ }^{1}$ Department of Electronic Convergence Engineering, Kwangwoon University, Seoul 139-701, Republic of Korea \\ ${ }^{2}$ Agency for Defense Development, Daejeon 305-600, Republic of Korea
}

Correspondence should be addressed to Young-Seek Chung; yschung@kw.ac.kr

Received 13 October 2014; Accepted 5 February 2015

Academic Editor: Luciano Tarricone

Copyright (C) 2015 Kang-in Lee et al. This is an open access article distributed under the Creative Commons Attribution License, which permits unrestricted use, distribution, and reproduction in any medium, provided the original work is properly cited.

\begin{abstract}
We propose a new algorithm to suppress the jammer signals and estimate the direction of arrival (DOA) of the signal of interest (SOI) for collocated MIMO radar by using the matrix pencil method (MPM) and the generalized likelihood ratio test (GLRT). The conventional GLRT divides the visible region into small angle samples, suppresses the jammer signals at each angle sample, and then estimates the DOA of the SOI. In the proposed algorithm, we extract the eigenvalues of received signals regardless of the SOI and jammer by using the MPM, which contain the information of the DOA of SOIs or jammers. Then, in order to suppress the jammers, we apply the GLRT to the extracted DOAs instead of to the entire visible region. By applying the MPM again to the received signals in which the jammer signals are suppressed, we can estimate the DOAs of the SOI. Since the proposed algorithm does not depend on the number of angle samples, it shows fast and accurate results regardless of the angle resolution. In order to verify the proposed algorithm, we compared the results with the results of the conventional GLRT and show the computing time.
\end{abstract}

\section{Introduction}

In recent times, multi-input multioutput (MIMO) radar technologies have been of interest in many areas of military and civilians, and it has been verified that MIMO radar has a lot of potential advantages over conventional phased-array radar [1-8]. In military area, the MIMO radar has an important role of detecting and tracking the low-observable targets and performing high resolution imaging. In also commercial areas such as the ITS (intelligent transport system), the collision avoidance system of car, and the weather broadcasting radar, the MIMO radar has attracted considerable attention.

A MIMO radar system is composed of $M_{t}$ transmitters and $M_{r}$ receivers, which can be collocated or distributed spatially [3]. Typically, it is assumed that at each receiver a technique exists for unambiguously separating the reflected signals of interest (SOI) from each transmitter, by utilizing orthogonal waveforms and a matched filter bank.

In $[5,9]$, many researchers have shown that, by utilizing orthogonal waveforms, a MIMO radar system with spatially diverse transmitters and receivers, can provide advantages in target detection and parameter estimation compared to a traditional phased array system [10]. For collocated transmit and receive antennas, the MIMO radar has been shown to get higher resolution than that of a phased array radar using the same number of physical antenna elements. This is because the MIMO radar has more degrees of freedom (DOF) than a phased array system with a single transmitting element $[3,4]$. In general, the received signal of the radar is composed of the signal of interest (SOI), interference or jammer, and noise. In order to extract the SOI from the received signal, the jammer signal should be suppressed efficiently and various suppression algorithms have been studied [11-15].

In this paper, we propose a new jammer suppression algorithm for the MIMO radar by combining the MPM and the GLRT. The conventional GLRT divides the entire angle region or the visible region $\Omega$ into an angle sample $\Delta \theta=$ $\Omega / N_{\theta}$, where $N_{\theta}$ is the number of angle samples $[1,16-$ 20]. It then performs suppression of the jammer signals or estimation of the DOAs of the SOI, by comparison with the given threshold value at each angle sample. If the angle of the DOA is an integer multiple of the angle sample (which in this 
paper is called the matched angle), the performance of the conventional GLRT shows good results; otherwise, the results may be of relatively poor quality. Although we can solve this problem by using a smaller angle sample, it increases the computing time.

In this paper, we first extract the number and DOA of signals above a threshold by using MPM. The extracted signals can be SOIs or jammers. Then, the jammer signals can be suppressed, by applying the GLRT to the extracted DOAs. In general, since the number of the extracted signals is much smaller than $N_{\theta}$, the proposed algorithm can reduce the number of operations of the GLRT compared with the conventional algorithm. The DOAs of the SOI can then be estimated by applying the MPM again to the output signals of the virtual array of the MIMO radar.

In this paper, we verify the performance of the proposed algorithm under various environments. In the first case, we test it according to the level of the jammer-to-noise ratio (JNR). In the other case, when the DOAs of the signals match the angle sample or do not, we compare the results of the proposed algorithm with those of the conventional one. Since the proposed algorithm estimates the DOAs of all the signals regardless of the angle sample, it shows good performance even when the DOA is not matched to the angle sample.

Also, we derive the computational complexity of the proposed algorithm and compare it with that of the conventional one.

\section{Signal Model}

In this paper, we assume the following:

(i) the transmitted probing signal is assumed to be a narrowband signal [1, 21];

(ii) there is nondispersive propagation;

(iii) transmitters and receivers are collocated;

(iv) the targets are represented by nonmoving points.

In general, MIMO radar is composed of $M_{t}$ transmitters with orthogonal waveforms and $M_{r}$ receivers with matched filters. At the target location, the transmitted signals are given by

$$
\sum_{m=1}^{M_{t}} e^{-j \omega_{0} \tau_{m}(\theta)} x_{t, m}(n) \equiv \mathbf{a}^{H}(\theta) \mathbf{x}_{t}(n), \quad n=1, \ldots, N,
$$

where, $x_{t, m}(n)$ is the discrete-time baseband signal sequence from the $m$ th transmitter, $\theta$ is the azimuth angle of the target, $\omega_{0}$ is the carrier angular frequency of the radar, $\tau_{m}(\theta)$ is the propagation time from the $m$ th transmitter to the target, and $N$ is the number of data samples of the transmitted signal.

Also, $(\cdot)^{H}$ means the Hermitian transpose, $\mathbf{x}_{t}(n)$ the transmitted sequence vector, and $\mathbf{a}(\theta)$ the steering vector of the transmitting antenna, which are represented by

$$
\begin{aligned}
& \mathbf{x}_{t}(n)=\left[\begin{array}{llll}
x_{t, 1}(n) & x_{t, 2}(n) & \cdots & x_{t, M_{t}}(n)
\end{array}\right]^{T}, \\
& \mathbf{a}(\theta)=\left[\begin{array}{llll}
e^{-j \omega_{0} \tau_{1}(\theta)} & e^{-j \omega_{0} \tau_{2}(\theta)} & \cdots & e^{-j \omega_{0} \tau_{M_{t}}(\theta)}
\end{array}\right]^{T},
\end{aligned}
$$

where $[\cdot]^{T}$ means the transpose of a vector. At the receiver, the received signal sequence vector is given by

$$
\begin{aligned}
& \mathbf{y}(n)=\sum_{k=1}^{K} \beta_{k} \mathbf{b}^{*}\left(\theta_{k}\right) \mathbf{a}^{H}\left(\theta_{k}\right) \mathbf{x}_{t}(n)+\mathbf{J}(n)+\varepsilon(n), \\
& \mathbf{y}(n)=\left[\begin{array}{llll}
y_{1}(n) & y_{2}(n) & \cdots & y_{M_{r}}(n)
\end{array}\right]^{T}, \\
& \mathbf{b}(\theta)=\left[\begin{array}{llll}
e^{-j \omega_{0} \widehat{\tau}_{1}(\theta)} & e^{-j \omega_{0} \widehat{\tau}_{2}(\theta)} & \cdots & e^{-j \omega_{0} \widehat{\tau}_{M_{r}}(\theta)}
\end{array}\right]^{T},
\end{aligned}
$$

where $\widehat{\tau}_{n}(\theta)$ is the propagation time from the target to the $n$th receiver, $\theta_{k}$ is the $k$ th target location, $\beta_{k}$ is the complex amplitude proportional to RCS of the $k$ th target, $\varepsilon(n)$ is the spatially and temporally white circularly symmetric complex Gaussian noise sequence vector with mean zero [1], $K$ is the number of targets of interest, and $\mathbf{J}(n)$ represents the jamming signal vector. Also, $\mathbf{b}(\theta)$ is the steering vector of the receiving antenna and $(\cdot)^{*}$ denotes the complex conjugate of a vector.

In (3), the received jamming signal can be written as

$$
\begin{aligned}
& \mathbf{J}(n)=\sum_{j=1}^{P} \beta_{j} \mathbf{b}^{*}\left(\theta_{j}\right) x_{j}(n), \\
& \mathbf{J}(n)=\left[\begin{array}{lllll}
J_{1}(n) & J_{2}(n) & \cdots & J_{M_{r}}(n)
\end{array}\right]^{T},
\end{aligned}
$$

where $\theta_{j}$ is the $j$ th jammer location, $\beta_{j}$ the complex amplitude of the $j$ th jammer, and $x_{j}$ the discrete-time baseband random signal of the $j$ th jammer. $P$ denotes the number of jammer signals.

\section{Jammer Suppression and DOA Estimation Using MPM and GLRT}

The matrix pencil method (MPM) approximates a given data set or function with the sum of the complex exponential functions. Applying the MPM to a phased array, it can extract the DOAs and the number of SOIs [22-24].

Consider a uniformly spaced linear array (ULA) antenna. Then, the received signal at the $m$ th antenna element of the ULA can be represented as

$$
\begin{gathered}
p(m)=\sum_{k=1}^{K} A_{k} \exp \left(j \frac{2 \pi d(m-1)}{\lambda} \sin \theta_{k}\right)=\sum_{k=1}^{K} A_{k} z_{k}^{m}, \\
m=1, \ldots, M, \\
z_{k} \equiv \exp \left(j \frac{2 \pi d}{\lambda} \sin \theta_{k}\right),
\end{gathered}
$$

where $K$ is the number of SOIs, $\lambda$ the wavelength, $d$ the distance between the adjacent antenna elements, $A_{k}$ the complex amplitude, and $\theta_{k}$ the DOA of the $k$ th SOI. In this paper, $z_{k}$ is called a pole. In a phased array, the pole vector $\left\{z_{k}\right\}$ corresponds to the steering vector that includes the DOAs of received signals. The MPM estimates the pole vector $\left\{z_{k}\right\}$ by applying singular value decomposition (SVD) to the Hankel matrix of (7).

In this paper, by using the MPM, we extract the DOAs of signals greater than the threshold $\gamma_{\mathrm{MPM}}$ from the received 
signal vector in the MIMO radar. Note that the extracted signals may not include a few jammers or SOIs according to the jammer-to-noise ratio (JNR) level.

When (5) is substituted into (3), (3) can be rewritten as

$$
\begin{aligned}
\mathbf{y}(n)= & \sum_{k=1}^{K} \beta_{k}\left[\begin{array}{c}
e^{j\left(2 \pi \sin \theta_{k} / \lambda\right)(0)} \\
\vdots \\
e^{j\left(2 \pi \sin \theta_{k} / \lambda\right)\left(\left(M_{r}-1\right) d_{r}\right)}
\end{array}\right] \\
& \times\left[\begin{array}{c}
e^{j\left(2 \pi \sin \theta_{k} / \lambda\right)(0)} \\
\vdots \\
e^{j\left(2 \pi \sin \theta_{k} / \lambda\right)\left(\left(M_{t}-1\right) d_{t}\right)}
\end{array}\right] \mathbf{x}_{t}(n) \\
+ & {\left[\begin{array}{c}
e^{j\left(2 \pi \sin \theta_{j} / \lambda\right)(0)} \\
\vdots \\
\sum_{j=1}^{P}
\end{array} \beta_{j}\left[\begin{array}{c}
x_{j}(n)+\varepsilon(n), \\
e^{j\left(2 \pi \sin \theta_{j} / \lambda\right)\left(\left(M_{r}-1\right) d_{r}\right)}
\end{array}\right]^{*}\right.}
\end{aligned}
$$

where $d_{t}$ and $d_{r}$ mean the distance between adjacent antennas of the receiver and the transmitter, respectively. Note that in the ULA, $\tau_{m}(\theta)=\exp \left(j\left(2 \pi \sin \theta d_{t} / \lambda\right)(m-1)\right)$ and $\widehat{\tau}_{n}(\theta)=$ $\exp \left(j\left(2 \pi \sin \theta d_{r} / \lambda\right)(n-1)\right)$. From (9), we can derive the following Hankel matrix $\mathbf{Y}_{H}(n)$ at each data sample as [22]:

$$
\mathbf{Y}_{H}(n)=\left[\begin{array}{cccc}
y_{1}(n) & y_{2}(n) & \cdots & y_{L}(n) \\
y_{2}(n) & y_{3}(n) & \cdots & y_{L+1}(n) \\
\vdots & \vdots & \ddots & \vdots \\
y_{M_{r}-L+1}(n) & y_{M_{r}-L+2}(n) & \cdots & y_{M_{r}}(n)
\end{array}\right]
$$

In $(10), y_{p}(n)$ is the received signal at the $p$ th receiver with the $n$th data sample and $L$ means the pencil parameter and its size is about $1 / 3$ the size of (9). Using the block matrix of (10), we construct a new matrix as [23]

$$
\overline{\mathbf{Y}}_{H}=\left[\mathbf{Y}_{H}(1), \mathbf{Y}_{H}(2), \ldots, \mathbf{Y}_{H}(N)\right] \text {. }
$$

Applying the MPM to (11), we extract the pole $z_{q}$ of the received signals above the threshold value as

$$
z_{q} \equiv \exp \left(j \frac{2 \pi d_{r}}{\lambda} \sin \theta_{q}\right), \quad q=1,2, \ldots, Q,
$$

where $\theta_{q}$ is the $q$ th DOA of the estimated signals and $Q$ is the number of the extracted poles. Using extracted pole $z_{q}$, the received signals can be reconstructed as

$$
\begin{gathered}
\mathbf{y}(n)=\sum_{q=1}^{Q} A_{q}(n) \mathbf{b}\left(\theta_{q}\right), \\
\mathbf{b}\left(\theta_{q}\right)=\left[1, \exp \left(j \frac{2 \pi d_{r}}{\lambda} \sin \theta_{q}\right), \ldots,\right. \\
\left.\exp \left(j \frac{2 \pi d_{r}}{\lambda}\left(M_{r}-1\right) \sin \theta_{q}\right)\right]^{T} \\
=\left[1, z_{q}, \ldots, z_{q}^{M_{r}-1}\right]^{T} .
\end{gathered}
$$

In general, $Q \leq K+P$; and in the case of high JNR, $Q$ converges to $P$. In (13), the complex amplitude vector $\mathbf{A}(n)$ can be estimated by using the least square method (LSM) as

$$
\mathbf{A}(n)=\mathbf{Z}^{\dagger}(n) \mathbf{y}(n),
$$

where

$$
\begin{aligned}
\mathbf{A}(n) & =\left[A_{1}(n), A_{2}(n), \ldots, A_{\mathrm{Q}}(n)\right]^{T}, \\
\mathbf{Z}(n) & =\left[\begin{array}{cccc}
1 & 1 & \cdots & 1 \\
z_{1}(n) & z_{2}(n) & \cdots & z_{\mathrm{Q}}(n) \\
\vdots & \vdots & & \vdots \\
z_{1}^{M_{r}-1}(n) & z_{2}^{M_{r}-1}(n) & \cdots & z_{\mathrm{Q}}^{M_{r}-1}(n)
\end{array}\right] .
\end{aligned}
$$

In (15), $(\cdot)^{\dagger}$ means the pseudo inverse of a matrix.

Applying the GLRT at the estimated DOA $\theta_{q}$, we determine whether the signal from $\theta_{q}$ is a jammer or an SOI. In this paper, in order to suppress the jammer signal, we use the following equation as the GLRT [5]:

$$
\rho(\theta)=1-\frac{\mathbf{b}^{T}(\theta) \widehat{\mathbf{R}}_{y y}^{-1} \mathbf{b}^{*}(\theta)}{\mathbf{b}^{T}(\theta) \widehat{\mathbf{Q}}^{-1} \mathbf{b}^{*}(\theta)} .
$$

Equation (17) represents the probability of the existence of the SOI at the testing angle $\theta$. While in the conventional GLRT method, $\theta$ is the integer multiplication of the angle sample $\Delta \theta$, the proposed method makes it possible to be an arbitrary angle estimated by applying the MPM to (11). In (17), $\widehat{\mathbf{R}}_{y y}$ denotes the sample covariance matrix of the received signal $\mathbf{Y}=[\mathbf{y}(1), \mathbf{y}(2), \ldots, \mathbf{y}(N)]$ and $\widehat{\mathbf{Q}}$ the residual covariance matrix as follows $[1,5,25]$ :

$$
\widehat{\mathbf{Q}}=\widehat{\mathbf{R}}_{y y}-\frac{\mathbf{Y} \mathbf{X}^{H} \mathbf{a}(\theta) \mathbf{a}^{H}(\theta) \mathbf{X} \mathbf{Y}^{H}}{N^{2} \mathbf{a}^{H}(\theta) \widehat{\mathbf{R}}_{x x} \mathbf{a}(\theta)}
$$

If the extracted angle $\theta_{q}$ satisfies $\rho\left(\theta_{q}\right)<\gamma_{\mathrm{GLRT}}$, it is considered as the DOA of a jammer. Note that when the JNR is very high, the first step MPM may fail to extract the DOA of the SOI due to its eigenvalue being lower than the threshold $\gamma_{\mathrm{MPM}}$. In this case, the proposed method can reconstruct the DOA of the SOI, by applying the MPM again to the received signal vector in which the jammer signals are suppressed.

Since the DOAs of the jammer signal are classified, we can find the corresponding amplitude $A_{J}$ to jammer's DOA in (15). Using these poles and amplitudes, we can obtain the received signal vector without the jammer signals as

$$
\widetilde{\mathbf{y}}(n)=\mathbf{y}(n)-\mathbf{Z}_{J}(n) \mathbf{A}_{J}(n),
$$

where,

$$
\begin{aligned}
\mathbf{A}_{J}(n)= & {\left[\begin{array}{lccc}
A_{1}(n) & A_{2}(n) & \cdots & A_{P}(n)
\end{array}\right]^{T}, } \\
\mathbf{Z}_{J}(n) & =\left[\begin{array}{cccc}
1 & 1 & \cdots & 1 \\
z_{1}(n) & z_{2}(n) & \cdots & z_{P}(n) \\
\vdots & \vdots & & \vdots \\
z_{1}^{M_{r}-1}(n) & z_{2}^{M_{r}-1}(n) & \cdots & z_{P}^{M_{r}-1}(n)
\end{array}\right] .
\end{aligned}
$$


In (19), since $\widetilde{\mathbf{y}}(n)$ is composed of only SOIs without jamming signals, it can be rewritten as

$$
\widetilde{\mathbf{y}}(n)=\sum_{k=1}^{K} \beta_{k} \mathbf{b}^{*}\left(\theta_{k}\right) \mathbf{a}^{H}\left(\theta_{k}\right) \mathbf{x}_{t}(n)+\varepsilon(n) .
$$

By applying the same number of matched filters as transmitters to each receiver, we obtain the receiving sequence matrix:

$$
\widetilde{\mathbf{Y}}(n)=\sum_{k=1}^{K} \beta_{k} \mathbf{b}^{*}\left(\theta_{k}\right) \mathbf{a}^{H}\left(\theta_{k}\right) \mathbf{x}_{t}(n) \mathbf{x}_{t}^{H}(n)+\varepsilon(n) \mathbf{x}_{t}^{H}(n) .
$$

If we choose an orthonormal sequence for $\mathbf{x}_{t}(n)$, the matrix $\mathbf{x}_{t}(n) \mathbf{x}_{t}^{H}(n)$ becomes an identity matrix I. Also, we assume the noise $\varepsilon(n)$ to be uncorrelated to $\mathbf{x}_{t}(n)$.

In the case of a uniformly spaced linear array (ULA), (22) can be rewritten as the following:

$$
\begin{aligned}
\widetilde{Y} & \left(m_{t}, m_{r}\right) \\
& =\sum_{k=1}^{K} \beta_{k} \exp \left(-j \frac{2 \pi}{\lambda} \sin \theta_{k}\left(d_{r}\left(m_{r}-1\right)-d_{t}\left(m_{t}-1\right)\right)\right),
\end{aligned}
$$

where $m_{t}=1, \ldots, M_{t}$ and $m_{r}=1, \ldots, M_{r}$. Assuming $d_{t}=$ $M_{r} d_{r},(23)$ can be simplified as

$$
Y(m)=\sum_{k=1}^{K} \beta_{k} \exp \left(-j \frac{2 \pi d_{r}}{\lambda}(m-1) \sin \theta_{k}\right)
$$

where $m=1,2, \ldots, M_{t} M_{r}$.

Applying the second step MPM to (24), we can obtain the DOAs of the SOI. The reason why the proposed method needs two applications of the MPM is because the first step MPM may fail to extract the DOAs of the SOI under very high JNR. Suppressing the jammer signals discriminated by the GLRT method, the second step of MPM can successfully extract the DOAs of the SOI from (24).

\section{Computational Complexity}

In this section, we compare the computational complexity between the proposed method and the conventional GLRT method. Assuming that $N_{\theta}$ is the number of angle samples, the computational complexity of the conventional method is approximately given by

$$
\left(N M_{r} M_{t}+M_{r}^{2} N+M_{r}^{3}\right) N_{\theta} .
$$

The computational complexity of the proposed method depends on the computation of the singular value decomposition (SVD) for the MPM. Assuming that $L_{1}$ and $L_{2}$ are the pencil parameters of the first and second MPM, respectively,

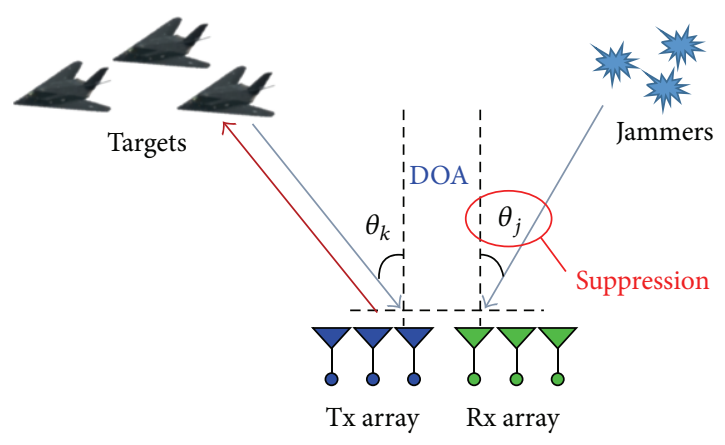

FIGURE 1: Simulation geometry for collocated MIMO radar signal model.

the computational complexity of the proposed method is approximately given by [26]:

$$
\begin{aligned}
& 2\left(\left(M_{r} M_{t}-L_{2}+1\right) L_{2}^{2}+2 L_{2}^{3}\right) \\
& \quad+2\left(\left(M_{r}-L_{1}+1\right)^{2} L_{1} N\right) \\
& \quad+\left(2\left(M_{r}-L_{1}+1\right)^{3}\right)+N\left(2 Q^{2} M_{r}+Q^{3}\right) .
\end{aligned}
$$

In this paper, we use $L_{1}=M_{r} / 3$ and $L_{2}=M_{t} M_{r} / 3$, and therefore the effect of $L_{2}$ is dominant. Figures 2 and 3 show a comparison of the computational complexity between the proposed method and the conventional GLRT method according to the number of virtual arrays $M_{t} M_{r}$ in the MIMO radar. For the conventional GLRT method, we use $\Delta \theta=$ $0.5^{\circ}$. In Figure 2, when $N=64$ and $M_{t} M_{r}<500$, the computational complexity of the proposed method is smaller than that of the conventional method. Figure 3 shows that when $N=128$ and $M_{t} M_{r}<700$, the complexity of the proposed method is smaller.

Therefore, the proposed algorithm is effective when the number of the virtual arrays in the MIMO radar is limited according to the number of data samples $N$.

\section{Numerical Examples}

Figure 1 is simulation geometry for collocated MIMO radar. In this figure, $\theta_{k}$ means $K$ units of SOI's DOA, and $\theta_{j}$ means the DOA of the qth jammer signal. The $T x$ array and the $R x$ array have the same phase center and it is assumed that the array elements locate on the same straight line.

Table 1 shows the simulation conditions for the information of the SOI, the JNR, and so forth. In the case of A and $B$, the JNR is set to be the same as the SNR. In the case of C and $\mathrm{D}$, the JNR is set to be much greater than the SNR. Also, in the case of $\mathrm{A}$ and $\mathrm{C}$, the DOAs of the SOI match the angle sample. In the case of B and D, the DOAs of the SOI do not match the angle sample.

In this work, the MIMO radar is assumed to be composed of a uniformly spaced linear array of 31 elements for receivers and 7 elements for transmitters, respectively. We set the distances between adjacent elements as $d_{r}=0.5 \lambda$ and $d_{t}=$ $M_{r} d_{r}=15.5 \lambda$. Also, the SNR varies from $0 \mathrm{~dB}$ to $20 \mathrm{~dB}$. We adopt a Walsh sequence for the transmitter signal $\mathbf{x}(n)$, 
TABLE 1: Simulation conditions.

\begin{tabular}{lcccc}
\hline Case & $\begin{array}{c}\text { JNR } \\
(\mathrm{dB})\end{array}$ & $\theta_{k}$ & $\beta_{k}$ & $\theta_{j}$ \\
\hline A & 20 & $\left(-10^{\circ}, 10^{\circ}, 30^{\circ}\right)$ & $(4.0,2.0,3.5)$ & $\left(-20^{\circ}, 0^{\circ}, 20^{\circ}\right)$ \\
B & 20 & $\left(-10.38^{\circ}, 10.29^{\circ}, 30.16^{\circ}\right)$ & $(4.0,2.0,3.5)$ & $\left(-20^{\circ}, 0^{\circ}, 20^{\circ}\right)$ \\
C & 70 & $\left(-10^{\circ}, 10^{\circ}, 30^{\circ}\right)$ & $(4.0,2.0,3.5)$ & $\left(-20^{\circ}, 0^{\circ}, 20^{\circ}\right)$ \\
D & 70 & $\left(-10.38^{\circ}, 10.29^{\circ}, 30.16^{\circ}\right)$ & $(4.0,2.0,3.5)$ & $\left(-20^{\circ}, 0^{\circ}, 20^{\circ}\right)$ \\
\hline
\end{tabular}

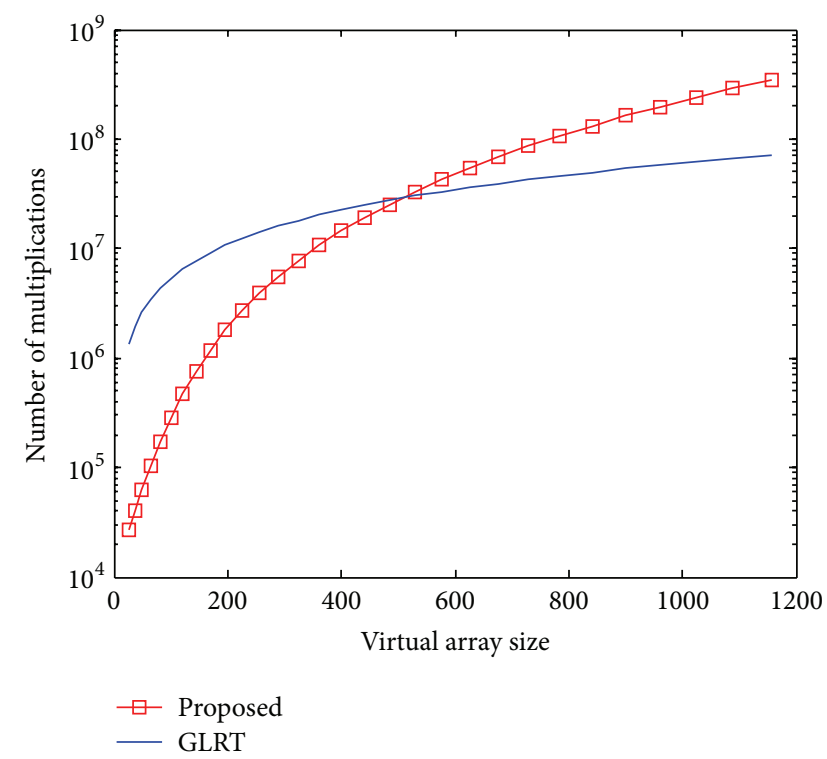

Figure 2: Computational complexity according to the size of the virtual array when $N=64$.

which has a covariance matrix of the form $\mathbf{R}_{x x}=\mathbf{I}$. We set $\gamma_{\mathrm{MPM}}=-10 \mathrm{~dB}$ and $\gamma_{\mathrm{GLRT}}=0.4$ for the threshold values. Also, for all examples, we use data samples with $N=128$ and 10,000 trials for the root mean square error (RMSE).

Figure 4 shows the normalized eigenvalues calculated by the first step MPM in the case of A and C. When the JNR and SNR have the same level, all of the jammer signals and the SOIs are shown to be above the threshold $\gamma_{\mathrm{MPM}}$. However, when the JNR is much greater than the SNR, only the jammer signals are shown to be above the threshold $\gamma_{\mathrm{MPM}}$.

Figure 5 shows the RMSE and the number of estimated SOIs with respect to the SNR in the case of A. Although the error of the proposed method is greater than that of the conventional GLRT method, the absolute magnitude of the error is low enough to be neglected. In this case, the two methods accurately estimate the number and the DOAs of the SOI.

Figure 6 shows the number of estimated SOIs with respect to the SNR in the case of B. In the case of using the conventional GLRT method, it fails to estimate the number of SOIs. This is because the DOA of the SOI does not match the angle sample. However, the proposed method successfully estimates the number of SOIs successfully. The RMSE of the proposed method is similar to that of case A.

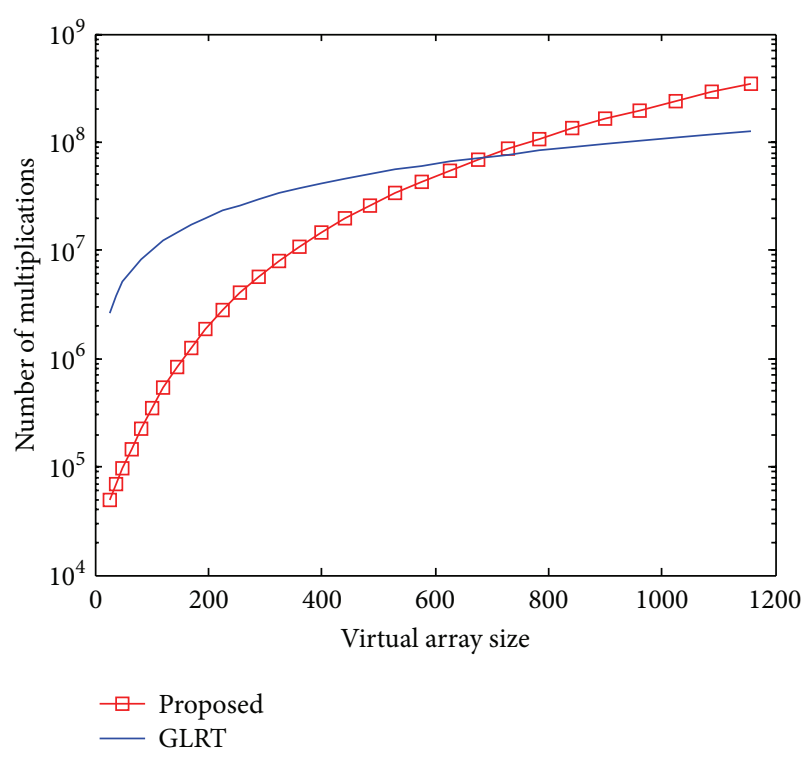

Figure 3: Computational complexity according to the size of the virtual array when $N=128$.

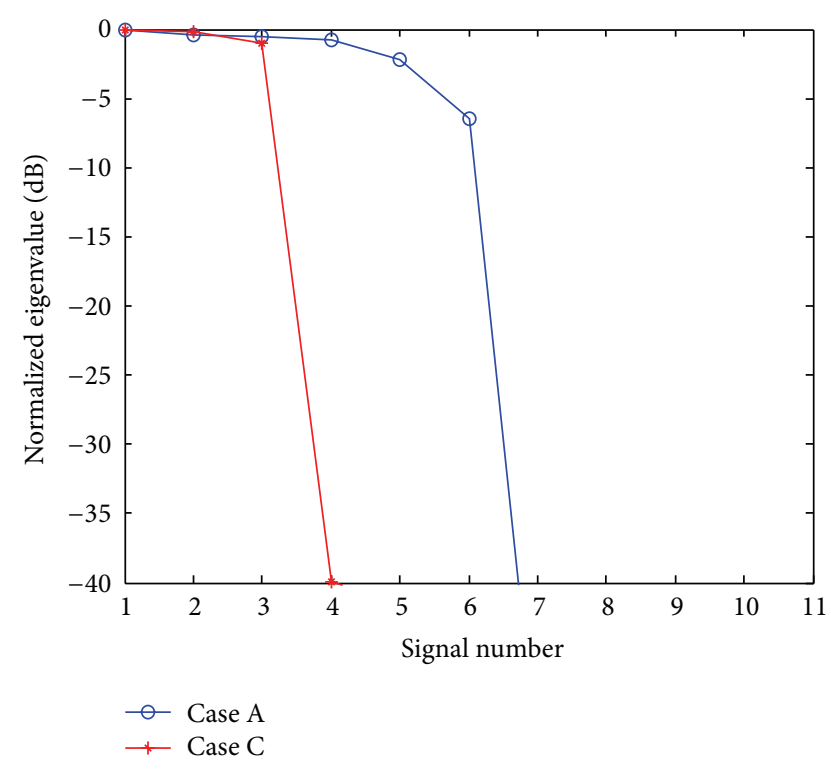

FIgURE 4: Normalized eigenvalues in the case of A and C, from the first step MPM.

Figure 7 shows the RMSE and the number of estimated SOIs with respect to the SNR in the case of C. In the proposed method, although the first step MPM fails to extract the DOAs of the SOI, the second step MPM can accurately extract them.

Figure 8 shows the number of estimated SOIs with respect to the SNR in the case of D. Since the DOA of the SOI does not match the angle sample, the results are similar to those of case B.

Figure 9 shows the number of SOIs estimated by the conventional GLRT method in the case of B, with respect to 


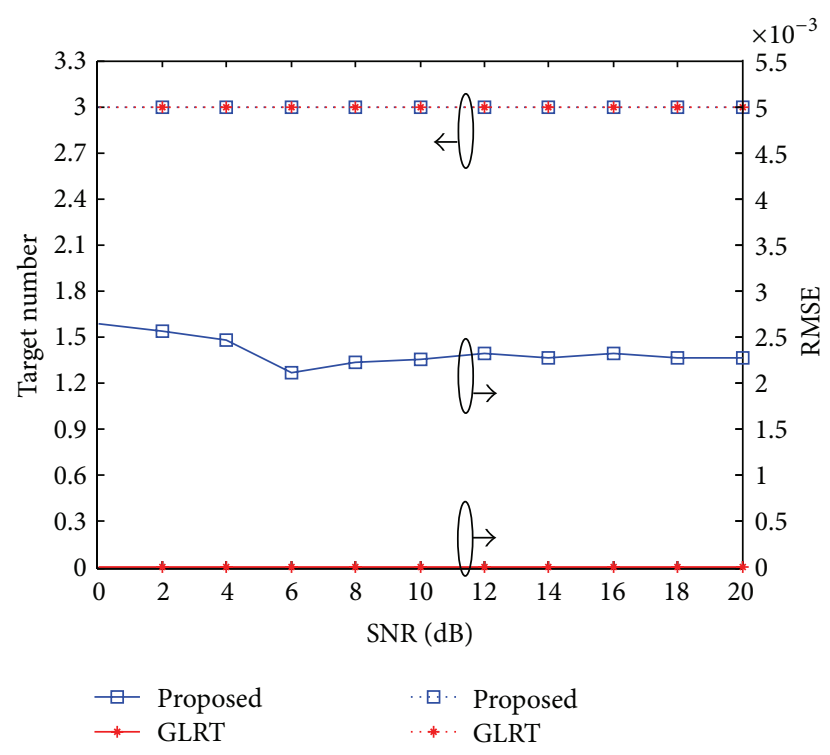

FIGURE 5: RMSE and the number of estimated SOIs in case A.

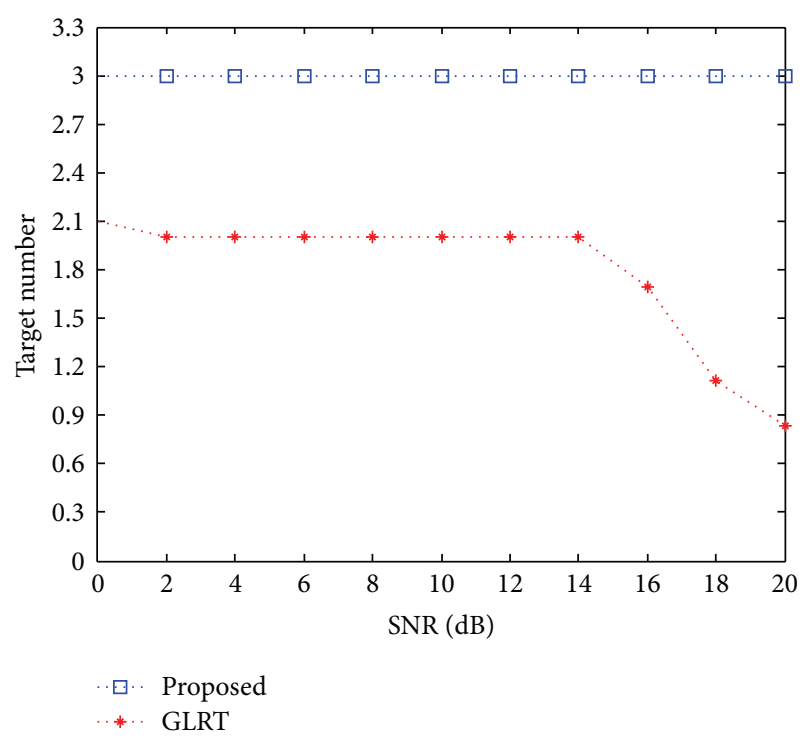

FIgURE 6: The number of estimated SOIs in case B.

the angle sample $\Delta \theta$. When the angle sample becomes small enough, the conventional method can estimate the DOAs of the SOI even if they do not match the angle sample. However, the computational complexity increases in proportion to the number of angle samples $N_{\theta}$.

From (25) and (26) under these simulation conditions, the computational complexity of the conventional method is about 8 times that of the proposed method. In these cases, after 10,000 trials on an Intel Core i7-3820 CPU @ 3.6 GHz, the average computing time of the proposed algorithm is $13.6 \mathrm{~ms}$ which is only $16 \%$ that of the conventional method of $84.3 \mathrm{~ms}$.

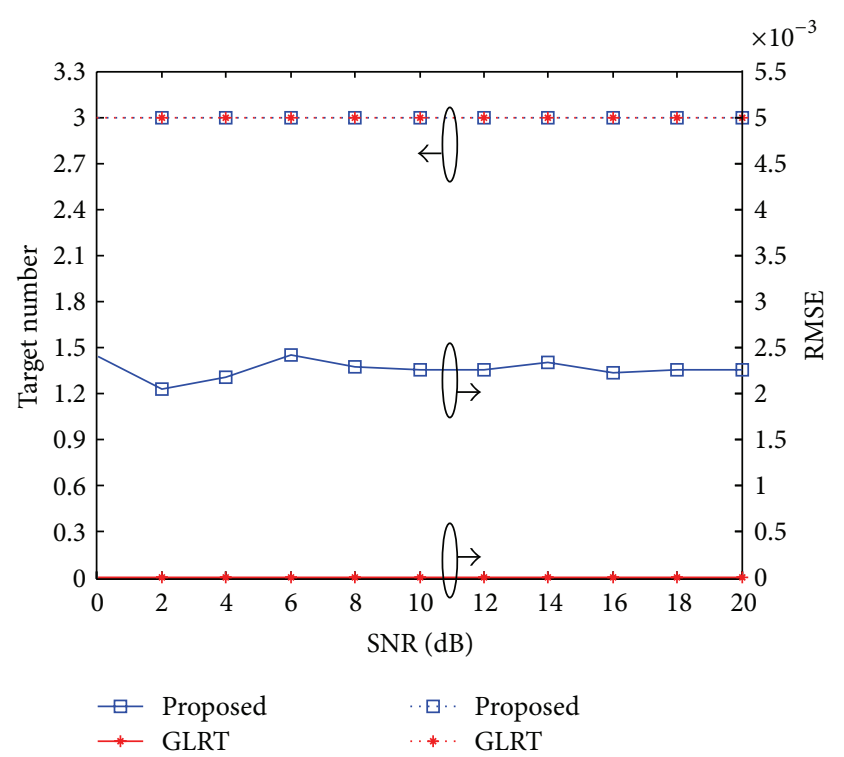

FIGURE 7: RMSE and the number of estimated SOIs, in case C.

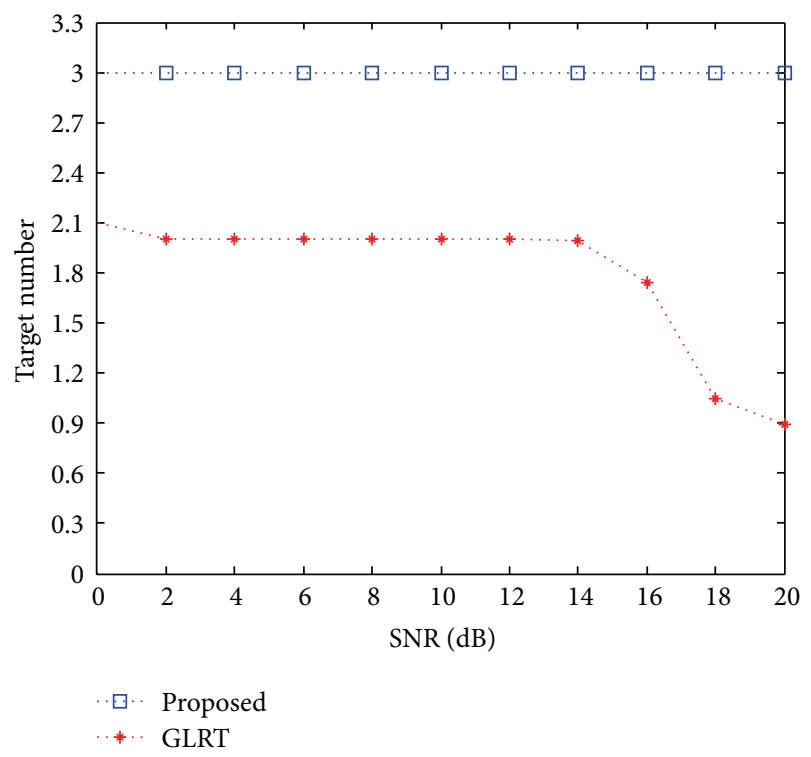

FIGURE 8: The number of estimated SOIs in case D.

\section{Conclusion}

In this paper, we propose a new jammer suppression technique for MIMO radar by exploiting the MPM and the GLRT. The jammer suppression is performed by preestimating the DOAs of the dominant signals above the threshold and applying the GLRT method to them. Although the proposed method may fail to classify a few DOA of the SOI at the first step MPM in the case of high JNR condition, it can accurately estimate the DOA of the SOI at the second step MPM. Also, the proposed method can estimate the DOAs of the SOI on a wide range of JNR and SNR independently of the angle sample, and the estimation error is negligible. In particular, 


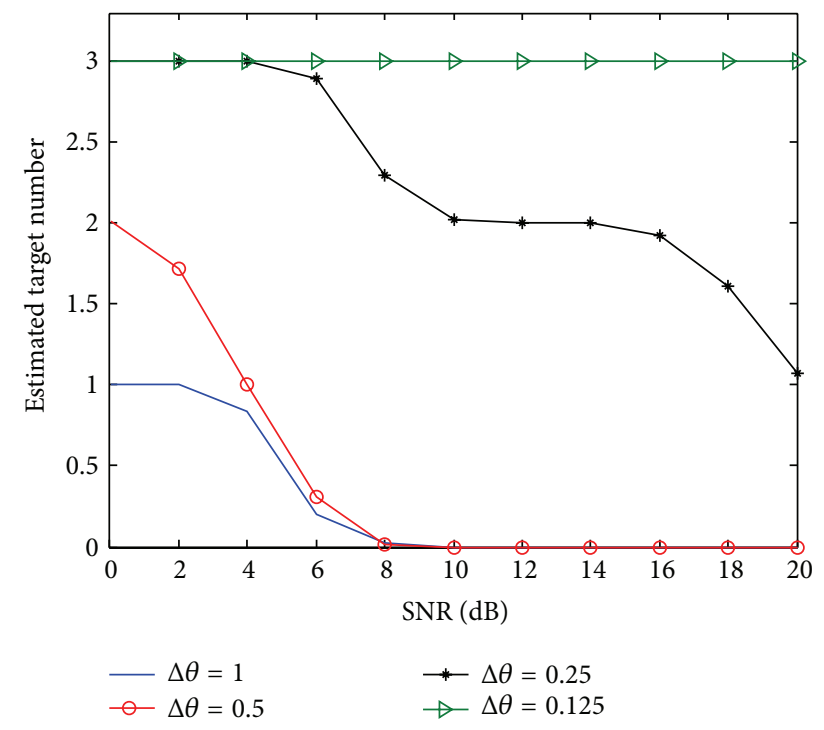

Figure 9: The number of estimated SOIs in case B, according to the size of the angle sample and the SNR.

in the case of the limited size of the virtual array in MIMO radar, the computational complexity can be reduced.

\section{Conflict of Interests}

The authors declare that there is no conflict of interests regarding the publication of this paper.

\section{Acknowledgments}

This work has been supported by the Low Observable Technology Research Center program of the Defense Acquisition Program Administration and the Agency for Defense Development.

\section{References}

[1] J. Li and P. Stoica, MIMO Radar Signal Processing, Wiley, 2008.

[2] E. Fishler, A. Haimovich, R. Blum, D. Chizhik, L. Cimini, and R. Valenzuela, "MIMO radar: an idea whose time has come," in Proceedings of the IEEE Radar Conference, pp. 71-78, April 2004.

[3] J. Li and P. Stoica, "MIMO radar with colocated antennas," IEEE Signal Processing Magazine, vol. 24, no. 5, pp. 106-114, 2007.

[4] J. Li and P. Stoica, "MIMO radar-diversity means superiority," in Proceedings of the 14th Annual Workshop on Adaptive Sensor Array Processing, MIT Lincoln Laboratory, Lexington, Mass, USA, 2006.

[5] L. Xu, J. Li, and P. Stoica, "Target detection and parameter estimation for MIMO radar systems," IEEE Transactions on Aerospace and Electronic Systems, vol. 44, no. 3, pp. 927-939, 2008.

[6] X. Wang, W. Wang, J. Liu, X. Li, and J. Wang, "A sparse representation scheme for angle estimation in monostatic MIMO radar," Signal Processing, vol. 104, pp. 258-263, 2014.
[7] R. Xie, Z. Liu, and Z.-J. Zhang, "DOA estimation for monostatic MIMO radar using polynomial rooting," Signal Processing, vol. 90, no. 12, pp. 3284-3288, 2010.

[8] W. Wang, X. Wang, H. Song, and Y. Ma, "Conjugate ESPRIT for DOA estimation in monostatic MIMO radar," Signal Processing, vol. 93, no. 7, pp. 2070-2075, 2013.

[9] J. Li, P. Stoica, L. Xu, and W. Roberts, "On parameter identifiability of MIMO radar," IEEE Signal Processing Letters, vol. 14, no. 12, pp. 968-971, 2007.

[10] H. Wang, G. Liao, Y. Wang, and X. Liu, "On parameter identifiability of MIMO radar with waveform diversity," Signal Processing, vol. 91, no. 8, pp. 2057-2063, 2011.

[11] A. Farina, "Electronic counter-countermeasures," in Radar Handbook, M. Skolnik, Ed., chapter 24, McGraw-Hill, New York, NY, USA, 3rd edition, 2008.

[12] S. Applebaum, "Adaptive arrays," IEEE Transactions on Antennas and Propagation, vol. 24, no. 5, pp. 585-598, 1976.

[13] U. Nickel, "Array processing for radar: achievements and challenges," International Journal of Antennas and Propagation, vol. 2013, Article ID 261230, 21 pages, 2013.

[14] M. Greco, F. Gini, and A. Farina, "Radar detection and classification of jamming signals belonging to a cone class," IEEE Transactions on Signal Processing, vol. 56, no. 5, pp. 1984-1993, 2008.

[15] G. de Vito, A. Farina, R. Sanzullo, and L. Timmoneri, "Comparison between two adaptive algorithms for cancellation of noise like and coherent repeater interference," in Proceedings of the International Symposium on Radar (IRS '98), Munich, Germany, September 1998.

[16] S. Z. Kalson, "An adaptive array detector with mismatched signal rejection," IEEE Transactions on Aerospace and Electronic Systems, vol. 28, no. 1, pp. 195-207, 1992.

[17] C. D. Richmond, "Performance of the adaptive sidelobe blanker detection algorithm in homogeneous environments," IEEE Transactions on Signal Processing, vol. 48, no. 5, pp. 1235-1247, 2000.

[18] O. Besson, "Detection of a signal in linear subspace with bounded mismatch," IEEE Transactions on Aerospace and Electronic Systems, vol. 42, no. 3, pp. 1131-1139, 2006.

[19] A. Coluccia and G. Ricci, "A radar network based W-ABORT approach to counteract deceptive ECM signals," in Proceedings of the IEEE International Symposium on Innovations in Intelligent Systems and Applications (INISTA '14), pp. 413-418, Alberobello, Italy, June 2014.

[20] A. Coluccia and G. Ricci, "A tunable W-ABORT-like detector with improved detection vs rejection capabilities trade-off," IEEE Signal Processing Letters, vol. 22, no. 6, pp. 713-717, 2015.

[21] H. L. van Trees, Optimum Array Processing, John Wiley \& Sons, New York, NY, USA, 2002.

[22] S. Burintramart, Methods of direction of arrival estimation using a single snapshot of the data [Ph.D. thesis], Department of Electrical Engineering, Syracuse University, Syracuse, NY, USA, 2009.

[23] E. L. Statzer, Matrix Pencil Method for Direction of Arrival Estimation with Uniform Circular Arrays, University of Cincinnati, Cincinnati, Ohio, USA, 2011.

[24] T. K. Sarkar and O. Pereira, "Using the matrix pencil method to estimate the parameters of a sum of complex exponentials," IEEE Antennas and Propagation Magazine, vol. 37, no. 1, pp. 4855, 1995. 
[25] W.-D. Wirth, Radar Techniques Using Array Antennas, The Institution of Engineering and Technology, 2013.

[26] G. H. Golub and C. F. Van Loan, Matrix Computations, vol. 3, The Johns Hopkins University Press, Baltimore, Md, USA, 2012. 

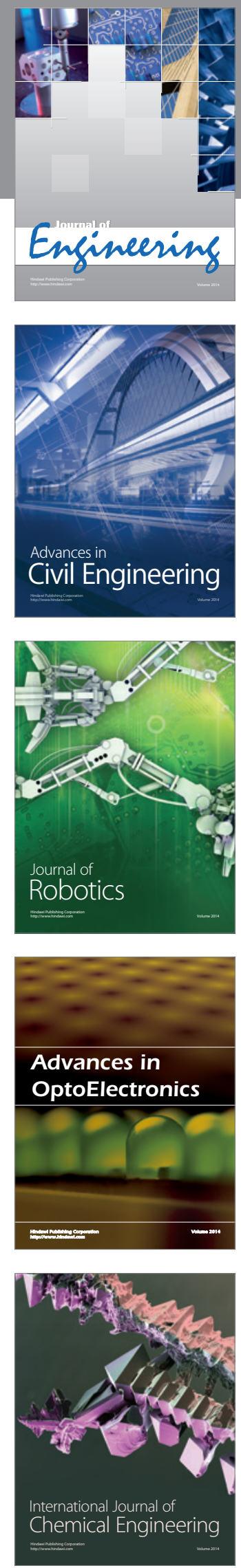

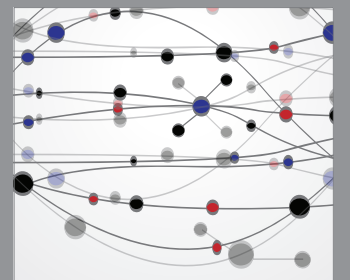

The Scientific World Journal
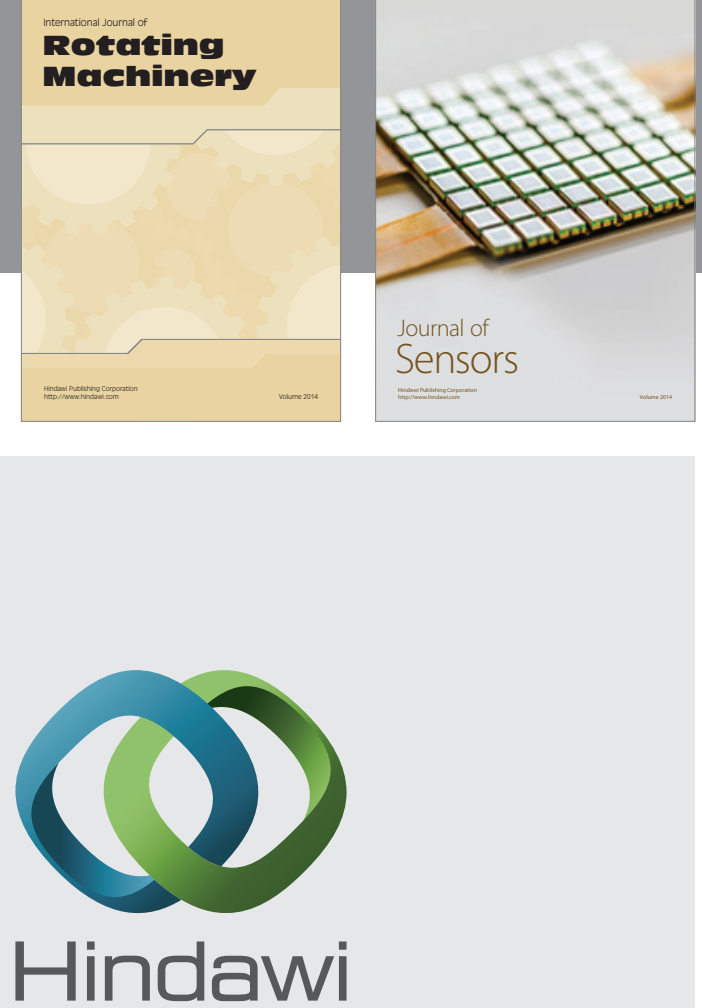

Submit your manuscripts at http://www.hindawi.com
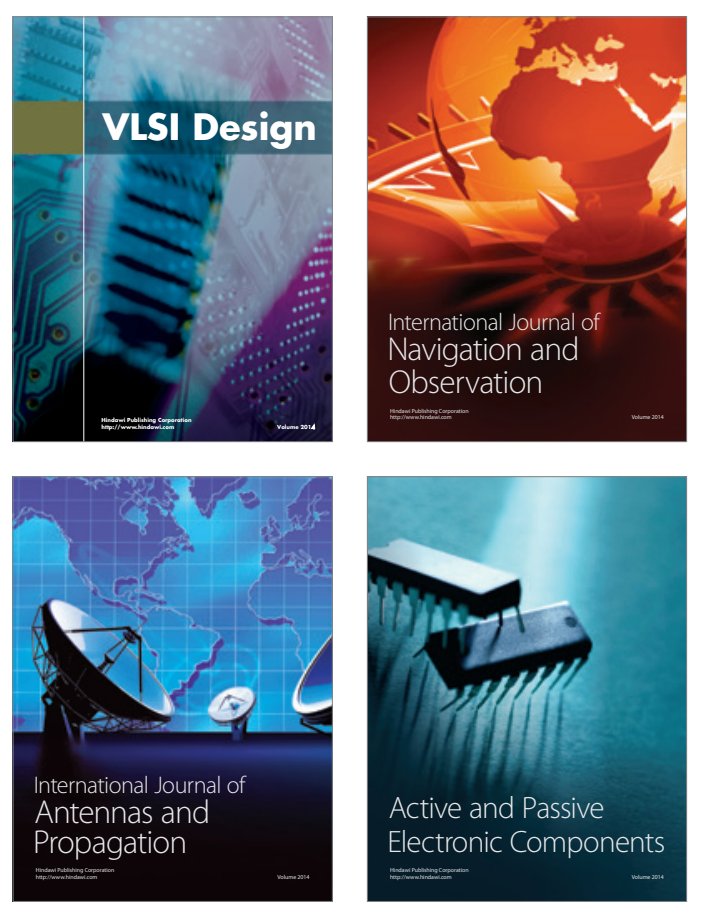
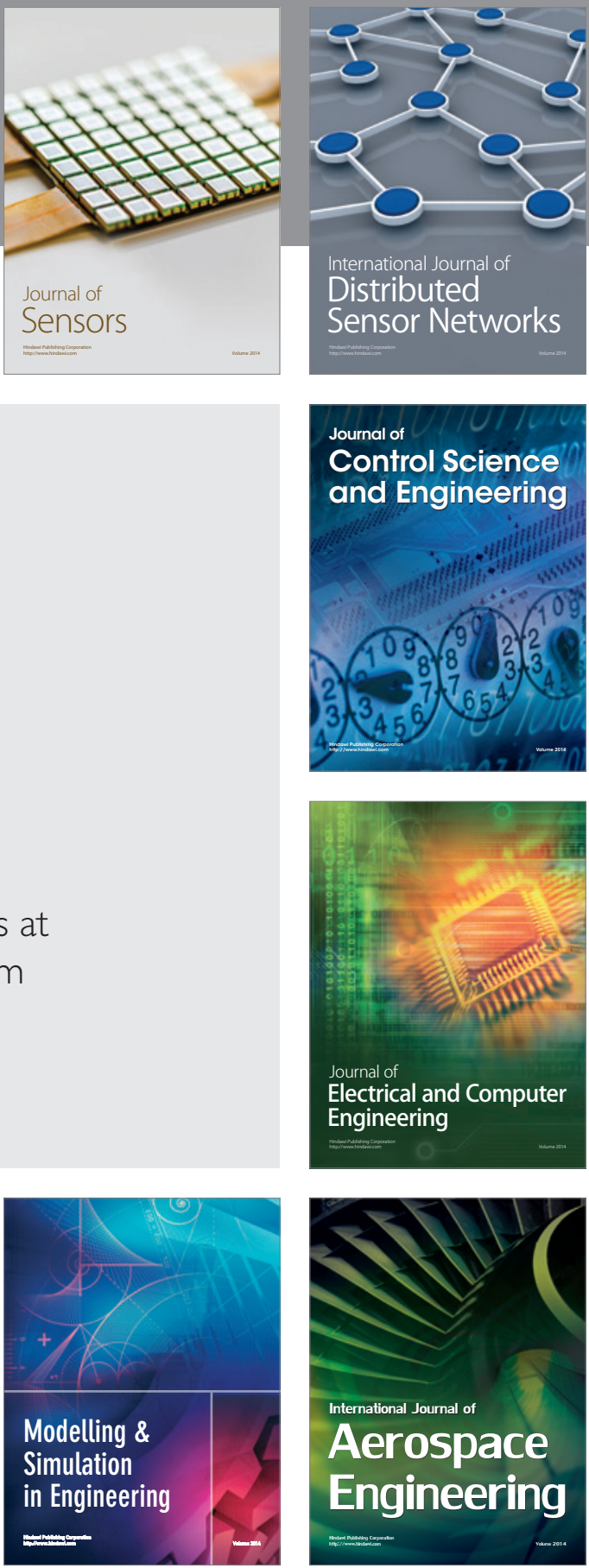

Journal of

Control Science

and Engineering
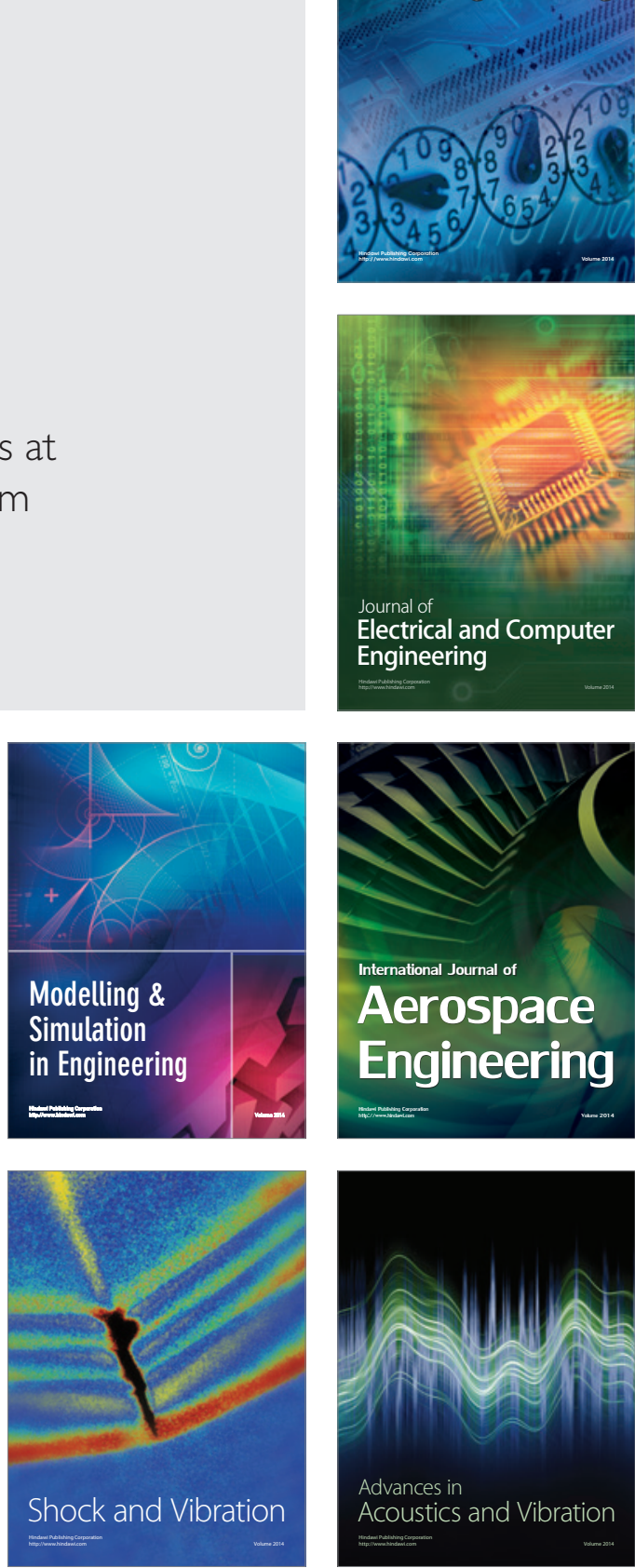